\title{
Psoriasis pustulosa generalizada: de la inmunopatogénesis a la clínica
}

\author{
Andrea Castañoㅁ, Margarita María Velásquez² \\ 1. Médica, residente de Dermatología, Universidad de Antioquia, Medellín, Colombia \\ 2. Médica dermatóloga, Ph.D. en Ciencias Básicas Biomédicas; docente de Dermatología, Universidad de Antioquia; Centro de \\ Investigaciones Dermatológicas CIDERM, Medellín, Colombia.
}

\section{RESUMEN}

El síndrome DITRA (Interleukin-36-Receptor Antagonist Deficiency) es una enfermedad autoinflamatoria debida a mutaciones del gen IL36RN que producen deficiencia del antagonista del receptor de la IL-36, lo que induce una cascada inflamatoria que lleva a un cuadro clínico grave de psoriasis pustulosa generalizada. Ante desencadenantes externos, como los componentes de agentes infecciosos que son activadores de los receptores de inmunidad innata, denominados PAMP (Pathogen-Associated Molecular Patterns), o los asociados a estrés celular, llamados DAMP (Damage-Associated Molecular Patterns), se activa el receptor con gran homología a los TLR (Toll-Like Receptors) y se amplifica a través del receptor de la IL-36, que en ausencia de su antagonista, conlleva a mayor activación celular en el principal órgano blanco, que es la piel.

El síndrome DITRA debe sospecharse en todo paciente con psoriasis pustulosa generalizada grave, para enfocar apropiadamente su tratamiento.

PAlabRas CLAVE: deficiencia del antagonista del receptor de la interleucina 36; interleucina 36; receptor de interleucina 36; proteína IL-36Ra; proteína L-36 ${ }^{\mathrm{a}}$; proteína IL-36B; psoriasis pustulosa, IL36RN; enfermedades autoinflamatorias hereditarias.

\section{SUMMARY}

The DITRA syndrome is an autoinflammatory disease due to mutations in the IL36RN gene causing deficiency of receptor's antagonist of IL-36, leading to an inflammatory cascade that causes clinical symptoms of generalized pustular psoriasis. External triggers such as components of infectious agents, which are activators of innate immune receptors, called PAMP (Pathogen-Associated Molecular Patterns) or associated with cellular stress (DAMP: Damage-Associated Molecular Patterns), activate the receptor highly homologous to the TLR (toll-like receptors) (1), and amplified through the receptor to IL-36, in the absence of its antagonist leads to increased cell activation in the main target organ, which is the skin.

The DITRA syndrome should be suspected in any patient with severe generalized pustular psoriasis, to properly focus their treatment.

KEY WORDS: Deficiency of interleukin-36 receptor antagonist, interleukin 36,

\section{Correspondencia:}

Andrea Castaño

Margarita María Velásquez

Email:

andreacv29@gmail.com y

mmvelasquez@yahoo.com

Recibido: 28/07/2015

Aceptado: 21/022017

Conflictos de interés:

No se reportan conflictos de interés. 
interleukin-36 receptor, IL-36RN protein, IL-36A protein, IL-36B protein, pustulosis psoriasis, IL36RN, hereditary autoinflammatory diseases.

\section{INTRODUCCIÓN}

Las enfermedades autoinflamatorias son un nuevo grupo de enfermedades raras, caracterizadas por una reacción inflamatoria anormalmente aumentada, mediada por la activación del sistema inmunitario innato, que conduce a síntomas inflamatorios devastadores en varios sistemas ${ }^{(1-3)}$. Aunque comparten algunos rasgos con las enfermedades autoinmunitarias, muestran características únicas como la periodicidad, la falta de participación de la inmunidad adaptativa, la proclividad a ser hereditarias y la etiología monogénica en la mayoría de ellas, lo que brinda la posibilidad de identificar el gen causante ${ }^{(1)}$.

Con el desarrollo de las tecnologías innovadoras, se ha incrementado el conocimiento de las moléculas del sistema inmunitario innato involucradas en las mutaciones que causan las inmunodeficiencias primarias; en consecuencia, varias interleucinas se han identificado y caracterizado en los últimos años. La familia de citocinas IL-1 está conformada por 11 citocinas que controlan una compleja red de señales proinflamatorias que reaccionan a las infecciones. De estas, la subfamilia IL-36 es las más diversa y la menos caracterizada ${ }^{(4)}$. Su disfunción se relaciona con la psoriasis pustulosa generalizada conocida como síndrome DITRA, causado por la mutación del gen del antagonista del receptor de la IL-36 (IL36RN), que aumenta la activación celular. Las mutaciones de este gen fueron originalmente descritas en familias de Túnez e Inglaterra ${ }^{(5-7)}$.

Los síndromes autoinflamatorios hacen parte de las inmunodeficiencias primarias $\mathrm{y}$, según la clasificación más reciente, se dividen en dos grandes grupos: los relacionados con el inflamosoma y los no relacionados con él; en este último grupo, se encuentra el síndrome DITRA (Interleukin-36-Receptor Antagonist Deficiency) ${ }^{(8,9)}$. Según la fisiopatogenia, las enfermedades autoinflamatorias pueden tener múltiples etiologías. Pueden deberse a mutaciones en el antagonismo de las citocinas o a alteraciones en la señalización de sus receptores. Otras se definen por su efecto y modo de acción en el sistema inmunitario innato como, por ejemplo, la activación del NFKB (Nuclear Factor Kappalight-chain-enhancer of activated B cells); en otras, se implican trastornos en el plegamiento de las proteínas, en la señalización de citocinas, y en la activación del macrófago; y aún hay un grupo de origen desconocido (3).

Esta revisión se enfoca en los aspectos inmunopatogénicos y clínicos del síndrome DITRA, presentando los fundamentos de las citocinas IL-36, su producción y regulación.

\section{Biología de las citocinas IL-36 y sus}

\section{antagonistas}

Hace casi dos décadas se descubrieron nuevos miembros de la familia de la IL-1, los cuales ejercen roles importantes en la inmunidad innata ${ }^{(10-12)} \mathrm{y}$, también, tienen la capacidad de estimular la inmunidad adaptativa ${ }^{(4)}$. Según su función biológica, se les dio una designación individual en la cual los ligandos son IL-36a, IL-36 $\beta$ e IL-36y, agonistas del receptor heterodimérico IL-36R, identificado en 1996 como una proteína de 561 aminoácidos ${ }^{(13)} \mathrm{y}$ originalmente descrito en el cerebro; por su parte, el antagonista de este receptor es IL-36Ra. Estas moléculas son de aproximadamente $18 \mathrm{kDa}$ y se diferencian sólo por unos pocos aminoácidos en el extremo N-terminal, lo que explica las diferencias en la afinidad por el receptor ${ }^{(6,14)}$; tienen potente actividad inflamatoria y reclutan células a la piel, tales como linfocitos T, neutrófilos y células dendríticas mieloides ${ }^{(10)}$. Los genes que codifican estas citocinas están localizados en el brazo largo del cromosoma 2 (2q), muy cerca de los genes de la IL-1 $\alpha$, la IL-1 $\beta$ y la IL-1Ra, lo que sugiere un gen ancestral común ${ }^{(15,16)}$. Además, al receptor IL-36R también se unen la IL-1 $\alpha$ y la IL-1 $\beta^{(17)}$, y dos nuevas citocinas, la IL-1ס y la IL-1ع; esta última activa el complejo proteico NF-kB, mientras que la IL$1 \delta$, con homología notable con el antagonista de los receptores de IL-1, inhibe específica y potentemente la reacción de la IL-1ع, mediante el mismo receptor. La ex-

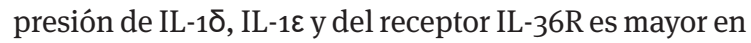
la piel con psoriasis que en la piel sana; esto podría sugerir que las tres constituyen un sistema de activación independiente en las barreras epiteliales, que participa en la reacción inflamatoria local ${ }^{(18)}$.

Por otro lado, el IL-36Ra es un antagonista del IL36R que bloquea su activación y señalización ${ }^{(15,16,19)}$; comparte $52 \%$ de homología con el antagonista del receptor de IL-1 (IL-1Ra) ${ }^{(10,20)}$, pero su actividad biológica es diferente. La IL-36 $\beta$ y la IL-36y comparten las características clásicas de los miembros de la familia IL-1, pero comparten $27 \%$ y $20 \%$ de homología con el antagonista IL-1Ra, respectivamente ${ }^{(15)}$. A pesar de los avances en el conocimiento de estas nuevas citocinas y de las mutaciones del antagonista IL-36Ra, su regulación y función aún son poco entendidas ${ }^{(21)}$. 


\section{IL-38}

La IL-38 es otra citocina de la familia IL-1 recientemente descubierta, se ha descrito que comparte $41 \%$ de homología con el antagonista IL-36Ra y $37 \%$ con el IL-1Ra, y que actúa como un antagonista contra el receptor IL-36R ${ }^{(4,22)}$. También, se sugiere que tanto la IL-38 como el IL-36Ra tienen actividad similar, porque ambos comparten tres características: incrementan al doble la IL-6 y la IL-4 ${ }^{(7)}$ inducidas por lipopolisacáridos en células dendríticas derivadas de monocitos, son antagonistas parciales del receptor IL-36R y se unen únicamente a este ${ }^{(22)}$.

En la tabla 1 se resumen las principales características de las citocinas IL-36 y sus antagonistas.

\begin{tabular}{|c|c|c|}
\hline $\begin{array}{l}\text { Citocinas IL-36, } \\
\text { antagonistas y receptor }\end{array}$ & Funciones & $\begin{array}{l}\text { Células que } \\
\text { la producen }\end{array}$ \\
\hline $\begin{array}{l}\qquad \begin{array}{l}\text { IL-36a } \\
\text { IL-36ß } \\
\text { IL-36y }\end{array} \\
\text { Se diferencian por unos pocos } \\
\text { aminoácidos en el extremo } \\
\text { N-terminal, lo que explica las } \\
\text { diferencias en la afinidad por el } \\
\text { receptor }\end{array}$ & 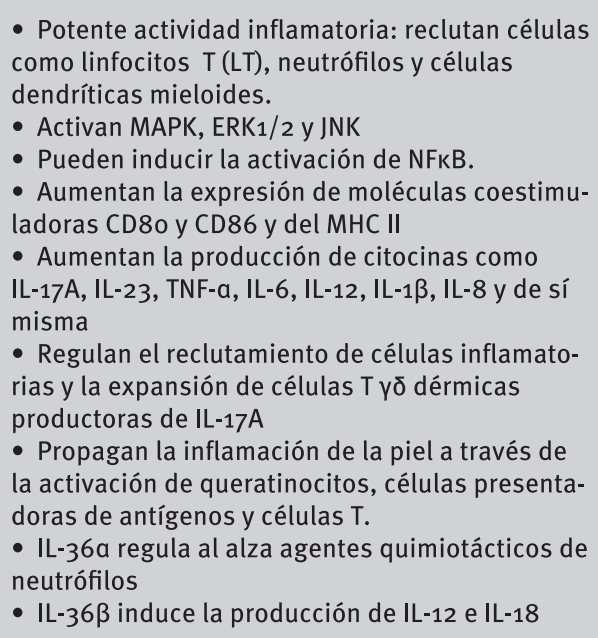 & 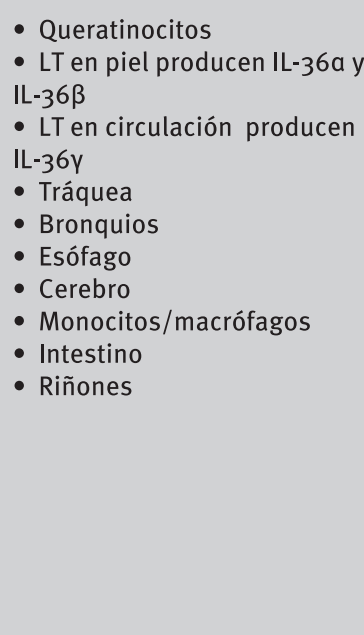 \\
\hline IL-36R & 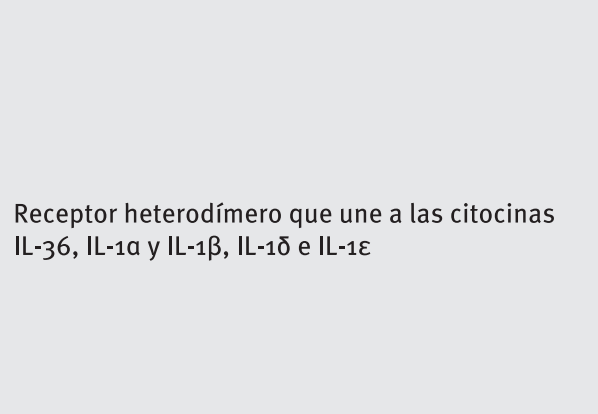 & $\begin{array}{l}\text { - Piel } \\
\text { - Tráquea } \\
\text { - Bronquios } \\
\text { - Esófago } \\
\text { - Cerebro } \\
\text { - Monocitos/macrófagos } \\
\text { - Intestino } \\
\text { - Riñones } \\
\text { - Células T CD4 vírgenes } \\
\text { - } \text { MDCs } \\
\text { - PDCs } \\
\text { - } \text { BMDCs }\end{array}$ \\
\hline IL-36Ra & $\begin{array}{l}\text { Antagonista de IL-36R que bloquea su activación } \\
\text { y señalización }\end{array}$ & Queratinocitos \\
\hline IL-38 & $\begin{array}{l}\text { comparte } 41 \% \text { de homología con IL-36Ra y } 37 \% \\
\text { con IL-1Ra, se ha descrito que actúa como un } \\
\text { antagonista para IL-36R }\end{array}$ & Queratinocitos \\
\hline
\end{tabular}

Tabla 1. Citocinas IL-36, sus antagonistas y su receptor. MDCs (células dendríticas derivadas de monocitos), PDCs (células dendríticas plasmocitoides), BMDCs (células dendríticas derivadas de la médula ósea) 


\section{Expresión de las citocinas IL-36 y su}

\section{receptor}

El receptor IL-36R, su antagonista IL-36Ra y sus ligandos se expresan principalmente en los queratinocitos de la piel con alteración de la barrera cutánea, y en otros epitelios expuestos a patógenos, tales como tráquea, bronquios y esófago; también, se expresan en cerebro, monocitos/macrófagos, intestino y riñones ${ }^{(14,17,23)}$. Además, los linfocitos T pueden expresar IL-36a e IL-36 3 , y en sangre periférica, IL-36Y ${ }^{(15-17)}$. Asimismo, la proteína accesoria del receptor de IL-1 (IL-1RAcP) es ampliamente expresada en la piel.

\section{Estructura de las citocinas IL-36 y su}

\section{receptor}

La estructura de IL-36y exhibe el pliegue típico $\beta$-trébol, consistente en 12 cadenas $\beta$ conectadas por 11 asas ${ }^{(4,24)}$. Este modelo muestra grandes diferencias de conformación entre la IL-36y y el antagonista IL-36Ra, y es probable que las asas $\beta 4 / 5$ y $\beta 11 / 12$ interactúen directamente con la proteína IL-1RAcP ${ }^{(4)}$.

La información disponible en humanos es limitada, pero Dunn, et al., describieron la estructura del antagonista IL-36Ra en ratón y, al compararla con la de la IL-1 $\beta$ y la IL-1Ra, encontraron el mismo pliegue trébol $\beta$. Sin embargo, el IL-36Ra posee dos estructuras helicoidales no encontradas en las otras, lo cual sugiere que son conformaciones nativas y que median en la unión específica al receptor. Además, existen grandes diferencias en cuanto a la longitud y la estructura en las asas que conectan las cadenas $\beta$, siendo ellas las que le confieren a la IL-1 $\beta$ y el IL-1Ra la especificidad de unión al receptor ${ }^{(25,26)}$.

Todos los receptores de la familia IL-1, incluyendo la subfamilia IL-36, comparten una estructura común con tres dominios extracelulares de inmunoglobulina, que tienen la capacidad de reconocer señales exógenas o endógenas de daño ${ }^{(13)}$. La dimerización del receptor de la IL-36 con la proteína accesoria, conduce a la señalización a través de los dominios intracitoplásmicos TIR (dominios homólogos al receptor toll-like) ${ }^{(13)}$; debido a esto, el receptor IL-36R es homólogo a los receptores de tipo toll (TLR) y desencadena una cascada de señalización intracelular similar a como lo hacen estos ${ }^{(26)}$.

\section{Procesamiento y secreción}

Las citocinas de la familia IL-1 requieren un procesamiento para ser plenamente activas, que consiste en la escisión de la metionina $\mathrm{N}$-terminal; esto hace que sean 1.000 a 10.000 veces más afines al receptor y, por lo tanto, más activas. Sin embargo, las proteasas responsables de esto se desconocen ${ }^{(15,19)}$.

Debido a que la IL-36a, la IL-36 $\beta$ y la IL-36y carecen de una secuencia de señal convencional, no pueden ser dirigidas al retículo endoplásmico y, por lo tanto, requieren mecanismos alternativos que les permitan ser secretadas. Aunque se presume que actúan extracelularmente, no es claro cómo son secretadas ${ }^{(14)}$; se sugiere que son externalizadas en forma dependiente de un estímulo comparable a IL-1 $\beta$, tal como el LPS/ATP (lipopolisacárido/trifosfato de adenosina), el cual es usado como un patrón molecular asociado al patógeno (Pathogen-Associated Molecular Patterns, PAMP) o al daño (Damage-Associated Molecular Patterns, DAMP) (15,19).

\section{Señalización}

Debido a que las citocinas de la familia IL-36 deben reclutar la proteína IL-1RAcP en un complejo ternario de citocina más proteína del receptor más proteína accesoria para la señalización funcional, se ha especulado que los modos de activación y señalización inhibidora a través del receptor IL-36R son similares a la inhibición y activación bien caracterizada del IL-1R por la IL-1 $\beta$ y el antagonista IL-1Ra, respectivamente ${ }^{(4)}$.

Para la inhibición del IL-36R se requiere que el IL-36Ra se una a su porción extracelular, bloquee el reclutamiento del segundo receptor de la IL-1RAcP y evite la formación de un complejo de señalización funcional (19). La ausencia del IL-36Ra amplifica la producción de citocinas como reacción a eventos activadores, y puede llevar a un síndrome de reacción inflamatoria sistémica y muerte, reportada en cinco casos de septicemia ${ }^{(27)}$. Las vías principales de transducción de señales que los receptores TLR emplean para activar las células, son la llamada vía del NF-kB y la proteína de activación 1 (AP-1), las cuales estimulan la expresión de genes de citocinas inflamatorias (TNF e IL-1), quimiocinas (CCL2 y CXCL8) y moléculas de adhesión endotelial (selectina E) ${ }^{(26)}$.

Después de una estrecha asociación espacial, los dominios intracitoplasmáticos TIR sirven de andamio para una serie de interacciones entre proteínas que activan la señalización ${ }^{(28)}$. El complejo receptor terciario recluta moléculas adaptadoras intracelulares, lo cual conduce a la activación y la translocación al núcleo del factor de transcripción NF-kB, y a la activación de las vías de señalización mediadas por cinasas proteínicas activadas por mitógeno, MAPK (Mitogen-Activated Protein Kinase), como la p38, las JNK (c-Jun N-terminal 
Kinases) y las ERK (Extracellular Signal-Regulated Kinases) ${ }^{(4,24,29,30)}$.

\section{Efectos inmunológicos de la IL-36}

Las IL-36 aumentan la expresión de las moléculas coestimuladoras CD8o y CD86 (b7.1, b7.2), del complejo mayor de histocompatibilidad de clase II ${ }^{(31)}$, de citocinas proinflamatorias, como IL-17A, IL-23, TNF- $\alpha$, IL-6, IL-12, IL-1ß e IL-8, y de ellas mismas ${ }^{(21,32)}$, actuando de forma autocrina o paracrina, similar a como la hace la IL-1. Johnston, et al., demostraron que la IL-17 y el TNF actúan de forma sinérgica favorecida por la IL-22, lo que puede inducir la expresión de IL-36a, IL-36 $\beta$ e IL-36y en los queratinocitos, constituyendo un asa de retroalimentación positiva. Tortola, et al., en un experimento con ratones tratados con imiquimod tópico, un agonista de TLR7 inductor de psoriasis en pacientes vulnerables, encontraron que la IL-36a regula el reclutamiento de células inflamatorias y la expansión de células T үo dérmicas productoras de IL-17a ${ }^{(32,33)}$.

El receptor IL-36R es expresado abundantemente en células T CD4 vírgenes, a las cuales dirige, en sinergia con la IL-12, hacia reacciones Th1 ${ }^{(4,14,15)}$. Dicho receptor también se expresa en las células dendríticas derivadas de monocitos, células dendríticas plasmocitoides ${ }^{(15)}$, macrófagos y células dendríticas derivadas de la médula ósea (Bone Marrow-Derived Dendritic Cells, BMDC), a las cuales estimula en su maduración y función ${ }^{(6)}$.

Las células dendríticas humanas derivadas de monocitos reaccionan a las IL-36 $\beta$ e IL-36y aumentando su expresión de HLA-DR y del marcador de maduración CD83. Además, la IL-36 $\beta$ induce la producción de IL-12 e IL-18, lo cual resulta en la proliferación de linfocitos T productores de IFNy, y estimuladas con IL-36a, regulan al alza agentes quimiotácticos de neutrófilos, como CXCL1, CXCL2, CXCL8, CCL3, CCL5, y CCL20, el TNFa y la expresión de CD40 ${ }^{(15,17,21)}$.

En sangre periférica humana, las células mononucleares estimuladas con Candida albicans, un potente inductor de la reacción Th17, producen menos IL-17 e IL-22 en presencia del antagonista IL-36Ra y de la IL-38 (22). Se encontró que el receptor IL-36R juega un rol en la regulación de las reacciones Th1 y Th17 inducidas por Aspergillus fumigatus, y que la neutralización del IL-36Ra endógeno podría amplificar la reacción Th17 a Aspergillus ${ }^{(15,23)}$. En un modelo de inmunización en ratones, usando seroalbúmina bovina (Bovine Serum Albumin, BSA), se observó que la IL-36 $\beta$ puede actuar como un adyuvante de reacciones Th1 in vivo, completamente dependiente de la señalización del receptor IL-36R ${ }^{(15)}$. La inyección intradérmica de IL-36a resulta en la expresión de quimiocinas, infiltración de leucocitos y acantosis en piel de ratones, pero no hay una reacción directa de linfocitos $\mathrm{T}$ CD8+, ya que estos no poseen el IL-36R ${ }^{(15,17,21)}$.

Estos datos indican que las citocinas IL-36 propagan la inflamación de la piel mediante la activación de queratinocitos, células presentadoras de antígenos y células T. Los queratinocitos no son pasivos y pueden iniciar cascadas inflamatorias después de exponerse a estrés físico, radiación ultravioleta o infección ${ }^{(21)}$. Aunque la expresión del antagonista IL-36Ra por los queratinocitos estimulados por la IL-17 en pacientes con psoriasis no difiere de la que se presenta en los controles sanos, hay correlación de la expresión génica de IL-36 con la reacción Th17 y el aumento de la activación de MAPK y NFKB, en lesiones de piel psoriática en hu$\operatorname{manos}^{(17,34)}$.

\section{Mutaciones en IL36RN}

Recientemente se ha demostrado que algunas mutaciones compuestas, homocigotas y heterocigotas, en el gen IL36RN causan una enfermedad que amenaza la vida, llamada psoriasis pustulosa generalizada (Generalized Pustular Psoriasis, GPP), las cuales fueron identificadas en casos familiares y esporádicos de varias etnias ${ }^{\left({ }^{6}\right)}$. Dichas mutaciones llevan a la introducción de un codón de parada prematuro, a una mutación o a sustitución de aminoácidos, lo cual resulta en una proteína mal plegada, menos estable y mal expresada, con

\section{"Los queratinocitos no son pasivos y pueden iniciar cascadas inflamatorias después de exponerse a estrés físico, radiación ultravioleta o infección."}


menos afinidad por el receptor y, por lo tanto, incapaz de atenuar la inflamación mediada por el receptor IL$36 \mathrm{R}^{(15,23)}$, lo cual incrementa la expresión de IL-8 y el reclutamiento de neutrófilos en la piel ${ }^{3}$.

Un siglo después del primer caso de psoriasis pustulosa generalizada descrito por von Zumbusch, la causa de la enfermedad permanecía desconocida, hasta que Marrakchi, et al., reportaron el gen causante de la psoriasis pustulosa generalizada familiar en $2011^{(29)}$. Ellos obtuvieron la secuenciación génica de nueve familias tunecinas y hallaron una mutación sin sentido en IL36RN, en el cromosoma 2q13-q14.1, con un cambio de posición de prolina por leucina en la posición 27 (L27P) ${ }^{(12)}$. El gen IL36RN tiene nueve residuos diferentes donde ocurren las mutaciones; los que están expuestos en la superficie afectan las interacciones entre el receptor IL36R y su antagonista IL-36Ra, y los que están ocultos resultan en un plegamiento incorrecto del IL-36Ra con reducción en su estabilidad ${ }^{(4)}$.

Actualmente, se cree que tres genes (IL36RN, AP1S3 y CARD14) que codifican proteínas secretadas por los queratinocitos, son los causantes de la propensión genética en la psoriasis pustulosa generalizada ${ }^{(29,37)}$. Esto coincide con lo hallado por Keerman, et al., quienes secuenciaron el ARN de la piel de pacientes con psoriasis con lesiones y sin ellas, y de la piel de pacientes control, y encontraron que todos los genes que codifican las citocinas IL-36 presentaban sobreexpresión en la piel con lesiones psoriáticas, lo que indica una condición para el desarrollo de la erupción psoriática ${ }^{(32)}$.

Además, Onoufriadis, et al., mediante la secuenciación del exoma de cinco pacientes con psoriasis pustulosa generalizada, en un modelo de herencia autosómica recesiva, reportaron que algunas mutaciones del IL36RN pueden causar psoriasis pustulosa generalizada esporádica. Este estudio demostró que dichas mutaciones y, por lo tanto, la deficiencia del antagonista del receptor de IL-36 (DITRA), precedieron a la psoriasis pustulosa generalizada esporádica en cinco pacientes europeos sin historia familiar, un hombre adulto japonés y una psoriasis pustulosa generalizada en una familiar tunecina ${ }^{(27,29)}$.

Posteriormente, los investigadores se centraron en la frecuencia de las mutaciones de IL36RN en los pacientes con psoriasis pustulosa generalizada. Sugiura, et al., buscaron mutaciones en dos grupos de pacientes con psoriasis pustulosa generalizada en la población japonesa: en uno, no asociada con psoriasis vulgar y, en otro, precedida por psoriasis vulgar. Se analizaron 11 casos del primer grupo y 20 casos del segundo ${ }^{(29,38)}$. Los resultados demostraron que 9 de los 11 casos no asociados con psoriasis vulgar (82\%) tenían mutaciones en el IL36RN; mientras que solo 2 de los 20 casos asociados con psoriasis vulgar (10\%) las tenían ${ }^{(29)}$. Una tendencia similar fue observada en un estudio de Korber, et al., quienes reportaron mutaciones recesivas en el IL36RN en 6 de 13 pacientes con psoriasis pustulosa generalizada sin psoriasis vulgar (46\%), pero solo en 1 de 6 pacientes con psoriasis pustulosa generalizada y psoriasis vulgar (17\%). Asimismo, Li, et al., reportaron que 18 de 30 casos de psoriasis pustulosa generalizada de inicio pediátrico tenían mutación en c.115 + 6T >C (p.Arg10ArgfsX1), y 9 de 38 casos de inicio en la adultez tenían la misma mutación homocigota ${ }^{(39)}$. Esto indica que la mayoría de los casos de psoriasis pustulosa generalizada no asociada con psoriasis vulgar son causados por mutaciones en el IL36RN y la mayoría, según el estudio epidemiológico de Ohkawara, et al., se inician en la niñez ${ }^{(29,40)}$.

El mecanismo patogénico de cómo los individuos con mutaciones heterocigotas en IL36RN son afectados por la psoriasis pustulosa generalizada es incierto. La frecuencia del alelo c.115 $+6 \mathrm{~T}>\mathrm{C}$ en la población china es de 2,05 \%, lo que predice que aproximadamente uno de cada 2.400 individuos serán homocigotos para este cambio. Esto excede la prevalencia en Asia, la cual es aproximadamente de uno por 100.000 individuos, por lo que se requerirán estudios futuros para identificar individuos asintomáticos con un genotipo c.115 $+6 \mathrm{C} / \mathrm{c} .115+6 \mathrm{C}^{(41)}$. En la tabla 2 se resumen las mutaciones más frecuentes y las poblaciones en las que se han encontrado.

Sin embargo, Capon sugirió que algunas mutaciones en un segundo locus génico podría explicar el inicio de la enfermedad, debido a la baja tasa de presentación de la psoriasis pustulosa generalizada en los hermanos que podrían tener la mutación heterocigota de IL36RN ${ }^{(29,41)}$. Además, los genes relacionados con el estrés y los factores que controlan su expresión en cada paciente, pueden afectar la gravedad del fenotipo de la enfermedad; una mayor capacidad individual de regular al alza un gen antioxidante, protegería contra el estrés oxidativo. Del mismo modo, la capacidad genética de sistemas de degradación más potentes evitaría la acumulación de productos de desecho y elevaría el umbral para la inflamación, incluso, en presencia de actividad metabólica excesiva, o proteínas mutadas o mal plegadas ${ }^{(1)}$.

Hussain, et al., demostraron un efecto de dosis genética, mediante el cual el inicio de la psoriasis pustulosa generalizada se retrasa significativamente en sujetos con mutaciones monoalélicas, aunque estos tengan un gran riesgo de inflamación sistémica. Por lo tanto, los 
pacientes heterocigotos pueden requerir una exposición más prolongada o más intensa a los desencadenantes ambientales, para manifestar la enfermedad ${ }^{(42)}$. En pacientes de Puerto Rico, se encontró una mutación poco frecuente, con deleción en el cromosoma 2, tanto de IL1RN como de cinco genes adyacentes: IL36RN, IL36a, IL36ß, IL36y y IL1F10 o IL-38. La frecuencia estimada del alelo encontrado en esta deleción es de 1,3 $\%$; como esta mutación engloba los tres agonistas del receptor IL-36R, probablemente elimina el efecto deletéreo de la pérdida de IL-36RN ${ }^{(43)}$.

\section{IL-36 y enfermedad}

Dado que se cree que las citocinas IL-36 actúan de forma autocrina en las células de la piel, como los queratinocitos y fibroblastos ${ }^{(33)}$, y que además juegan un rol fundamental en la inducción y regulación de la inflamación ${ }^{(43)}$, no es sorprendente que en las enfermedades cutáneas se hayan explorado las asociaciones con estas citocinas ${ }^{(15)}$.

A pesar de que las citocinas IL-36 se encuentran en varios tejidos, hasta el momento solo se han descrito manifestaciones patológicas en la piel y los pulmones. En el tejido sinovial de pacientes con artritis, se encontraron IL-36a, IL-36R e IL-36Ra ${ }^{(17)}$; pero, en un estudio de ratones con artritis inducida experimentalmente, no se encontró que sus niveles tuvieran correlación con la seriedad de la enfermedad. La inyección de un anticuerpo anti-IL-36R no tuvo ningún efecto en el desarrollo y la gravedad de la artritis inducida por colágeno, ni en el daño estructural o la gravedad de la inducida por antígeno; la gravedad fue similar, tanto en ratones silvestres como en aquellos con deficiencia de IL-36R ${ }^{(14)}$. Además, la IL-36ß sérica de sujetos sanos, comparada con la hallada en casos de artritis reumatoide, no presentó diferencias ${ }^{(23)}$.

Por otra parte, en un estudio de Ramadas, et al., en ratones, para determinar el efecto que ejerce la IL-36a en los pulmones, se encontró que actúa induciendo la expresión de moléculas coestimuladoras CD80, CD86 y CD40 en las células dendríticas, lo que incrementa la activación y proliferación de las células T, y de esta manera, actúa como una citocina proinflamatoria en enfermedades pulmonares ${ }^{(45)}$.

En cuanto a las enfermedades cutáneas, en 1910, von Zumbusch describió el primer caso de psoriasis pustulosa generalizada, que puede aparecer con historia de una psoriasis vulgar previa o sin ella y se caracteriza por una erupción generalizada, con pústulas estériles y edema, ocasionalmente lengua geográfica y distrofia ungular, dolor en la piel, ardor, sudoración y prurito (figura 1), fiebre repentina alta de 40 a $42{ }^{\circ} \mathrm{C}$, malestar general, fatiga, anorexia, elevación de la PCR, leucocitosis, y alteraciones circulatorias, respiratorias o septicemia que pueden amenazar la vida. En un tercio de los pacientes, se desarrolla artritis, principalmente en las articulaciones interfalángicas distales, pero también en las sacroilíacas ${ }^{(5,7,27,46)}$.

También se encuentra disminución del calcio sérico como resultado de la hipoalbuminemia, disfunción hepática e hipovolemia que conduce a una azoemia prerrenal. Esta psoriasis es episódica, sus síntomas pueden requerir tratamiento prolongado o remitir espontáneamente, y suelen recurrir después de la exposición a factores precipitantes. En los niños, el pronóstico es generalmente muy bueno, a pesar de la morbilidad asociada con los episodios agudos ${ }^{(27)}$. Más del 70\% de los pacientes desarrollan la enfermedad durante la niñez, aunque puede presentarse a cualquier edad $^{(2,46)}$.

Los factores desencadenantes incluyen infección, embarazo, hipocalcemia asociada con hipotiroidismo y medicamentos ${ }^{(29,37)}$. En casi todos los afectados de nueve familias tunecinas, se desencadenó por infecciones $(n=14)$, suspensión del tratamiento con retinoides $(n=7)$, menstruación $(n=6)$ o embarazo $(n=4)^{(7)}$. En $85 \%$ de los pacientes, la psoriasis de placas típicas precede la aparición de la psoriasis pustulosa generalizada, pero no de los casos de síndrome DITRA. El 25\% de los niños con psoriasis pustulosa tienen historia familiar de psoriasis, lo que sugiere un fuerte componente genético. En adultos, esta enfermedad es mucho menos común que la psoriasis en placas y tiene una prevalencia estimada de o,1 \% en la población general. La psoriasis pustulosa y la psoriasis en placas tienen locus de propensión HLA muy distintos, lo que sugiere que son dos categorías de enfermedad genéticamente diferentes ${ }^{(27)}$.

Los fenotipos de DITRA podrían incluir psoriasis pustulosa generalizada, psoriasis pustulosa palmoplantar (Palmar Plantar Pustulosis, PPP), acrodermatitis continua de Hallopeau (Acrodermatitis Continua of Hallopeau, ACH) y una erupción por medicamentos similar a la psoriasis pustulosa generalizada, llamada pustulosis exantemática generalizada aguda (Acute Generalized Exanthematous Pustulosis, AGEP) (47), ya que las mismas mutaciones en el gen IL36RN se detectaron en pacientes con estos trastornos. No obstante, como no hay diferencia fenotípica entre los que tienen y los que no tienen la mutación, el análisis genético de estos casos es importante para el establecimiento y la aplicación de tratamientos específicos dirigidos a la señalización de la IL-36 ${ }^{(36)}$. 

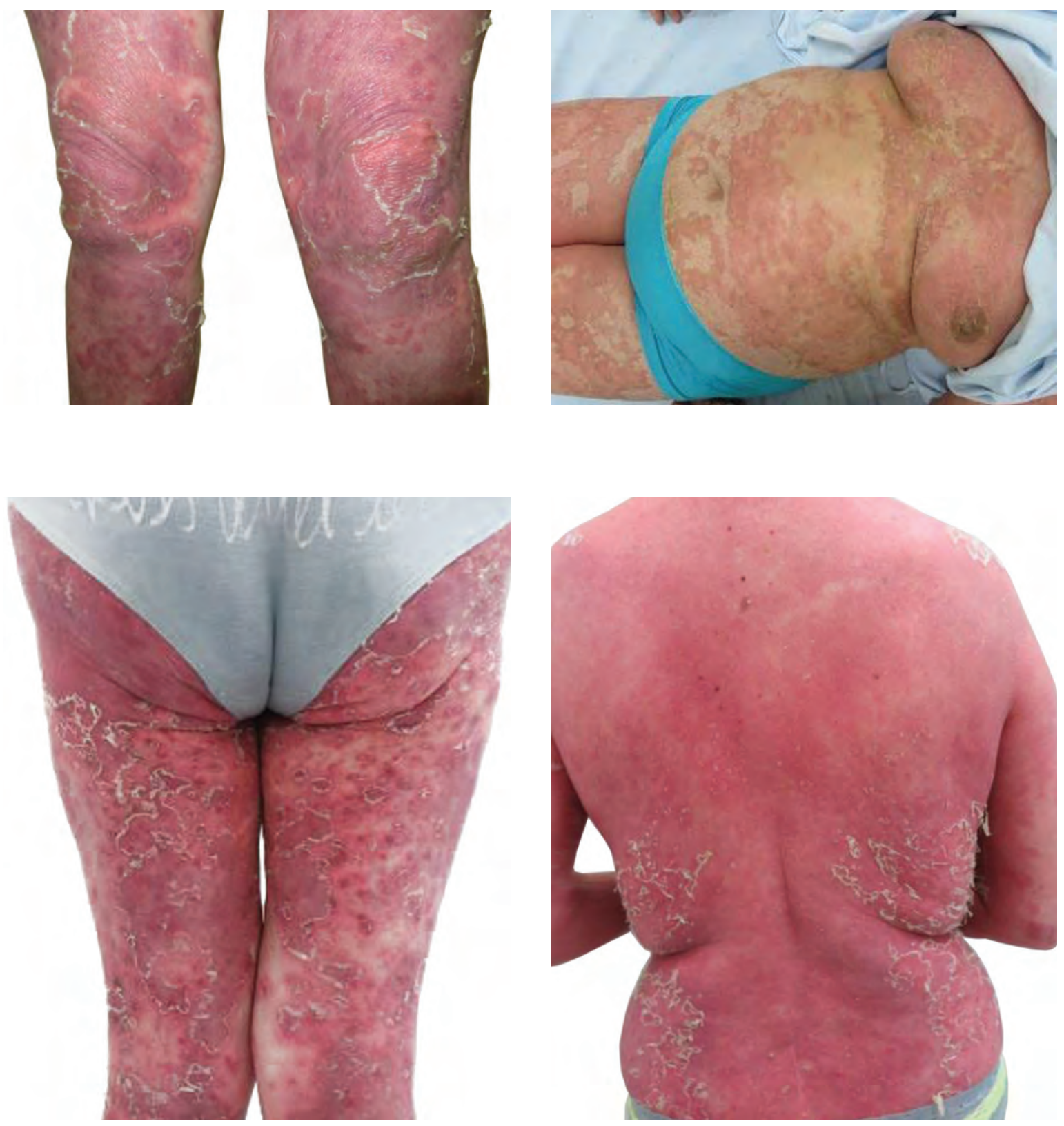

Figura 1. Hallazgos clínicos de psoriasis pustular generalizada. Piel intensamente inflamada con placas extensas de eritema y pústulas superpuestas, con abundante descamación generalizada, localizadas en tórax y extremidades. Fotos Sección de Dermatología, Facultad de Medicina, Universidad de Antioquia. 
Con respecto a un fenotipo específico, la acrodermatitis continua de Hallopeau es una erupción pustulosa, estéril y crónica, que afecta las yemas de los dedos y compromete las uñas; se han reportado casos que progresan a psoriasis pustulosa generalizada. Abbas, et al., realizaron una secuencia del exón 5 del gen IL36RN de un paciente masculino con acrodermatitis continua de Hallopeau y de su hermana que tenía historia de psoriasis pustulosa generalizada, e identificaron una mutación homocigota con sentido errado c.338C>T (p.Ser113Leu) en ambos pacientes. Según estos estudios, se evidenció que la acrodermatitis continua de Hallopeau y la psoriasis pustulosa generalizada se encuentran en el mismo espectro ${ }^{(10)}$. Además, uno de los pacientes del estudio de Marrakchi, et al., además de psoriasis pustulosa generalizada, presentaba signos de acrodermatitis continua de Hallopeau, lo que también apoya estos hallazgos ${ }^{(11)}$.

\section{Evidencias del papel de la IL-36 en el síndrome DITRA}

La relación de la IL-36 con el síndrome DITRA ha sido estudiada de manera amplia. En la tabla 3 se resume la evidencia experimental del papel de la IL-36, en especial, la mutación del antagonista del receptor que lleva al incremento en la función de esta citocina.

\section{TRATAMIENTO}

La mayoría de casos que presentan solo psoriasis pustulosa generalizada están asociados con el síndrome DITRA. Estos pacientes pueden ser tratados con esteroides tópicos, análogos de la vitamina D3 por vía tópica, ciclosporina A, acitretín, metotrexato, corticoides sistémicos, fotoquimioterapia PUVA, UVB de banda angosta, aféresis de granulocitos y monocitos (Granulocyte-Monocyte Apheresis, GMA) y terapias biológicas anti-TNFa, como adalimumab, etanercept e infliximab $(29,37,46)$.

Se ha investigado la eficacia de la aféresis de granulocitos y monocitos en la psoriasis pustulosa generalizada y fue indicada en el Japón, en 2012; además, Kazumitsu, et al., la reportaron como muy efectiva en un caso de DITRA ${ }^{(29)}$. Puede usarse como tratamiento alterno o adyuvante en algunos casos de psoriasis pustulosa generalizada, cuando los tratamientos anteriores no fueron efectivos o aplicables, en casos pediátricos o ancianos, en embarazadas, en infectados por el virus de la hepatitis $\mathrm{B} u$ en otras condiciones que limiten el uso de productos biológicos o inmunosupresores ${ }^{(37)}$. Chiharu, et al., reportaron una paciente de 78 años con DITRA, que fue tratada exitosamente con aféresis de granulocitos y monocitos. Inicialmente, fue tratada con prednisolona oral y ciclosporina, sin éxito terapéutico. Una biopsia reveló infiltrados inflamatorios en la piel, con una pústula espongiforme de Kogoj, indicativa de psoriasis pustulosa generalizada. Se encontró

\begin{tabular}{|lll|}
\hline MUTACIÓN & TIPO DE MUTACIÓN & POBLACIONES DONDE SE HA ENCONTRADO \\
\hline p.Arg10x & Sin sentido & Japón, Palestina \\
\hline p.Arg10ArgfsX1 & Sin sentido & Japón, China y Malasia \\
\hline p.Leu27pro & En sentido errado & África \\
\hline p.Lys35Arg & En sentido errado & Europa \\
\hline p.Arg48Trp & En sentido errado & Europa \\
\hline p.Arg102Trp & En sentido errado & Europa \\
\hline p.Ser113Leu & En sentido errado & Europa \\
\hline p.Thr123Arg & En sentido errado & El más frecuente en Europa \\
\hline Thr123.Met & En sentido errado & Sin dato \\
\hline Val57lle & Sin dato & Japón \\
\hline p.Pro82Leu & Sin dato & China \\
\hline p.E112K & Sin sentido & China \\
\hline
\end{tabular}

Tabla 2. Mutaciones conocidas en IL-36RN y las poblaciones donde se han encontrado. 
La expresión de IL-36R, IL-36Ra y IL-36a está aumentada en piel

Blumberg et al.

14 psoriática humana

Expresión transgénica de IL-36a en queratinocitos basales murinos resulta en alteraciones en la dermis y la epidermis

La gravedad de las anormalidades de la piel se incrementó en los

Blumberg et al. ratones deficientes de IL-36Ra (hiperqueratosis, acantosis, reclutamiento de neutrófilos y expansión de células T productoras de IL-17).

El IL-36R es fundamental en la inflamación de la piel psoriática humana trasplantada a ratones inmunodeficientes.

Blumberg et al.

Tortola et al.

Los ratones deficientes de IL-36R no desarrollaron la patología; demostrando que el balance endógeno entre ligandos del IL-36R y el IL-36Ra, juega un rol esencial para la salud de la piel

En nueve ratones transgénicos se expresó IL-36a en queratinocitos basales, el que tenía la más alta expresión presentó anormalidades severas en piel y murió.

Blumberg et al.

La histología demostró acantosis, hiperqueratosis y un infiltrado de

Blumberg et al. células inflamatorias en la dermis como neutrófilos, macrófagos y linfocitos.

Se demostró in vivo que IL-1RAcP y IL-36R son requeridos para la señalización de las tres citocinas IL-36, ya que anticuerpos dirigidos contra IL-1RAcP y la deficiencia de IL-36R, bloquean la activación de la vía que conduce a NF-KB y evitan las lesiones en piel.

Para determinar el papel deTNF-a, usaron un anticuerpo neutralizante contra TNF-a murino, demostrando una disminución significativa en el espesor epidérmico y en la cantidad de infiltrado inmune dérmico, comparado con las crías tratadas con un anticuerpo control.

Para entender la función de los neutrófilos, usaron en las crías el anticuerpo anti-Gr1 para la depleción de neutrófilos; la mayoría fueron extinguidos, lo cual disminuyó la severidad de la inflamación.

Towne et al.

30

La combinación de un promotor como K14 con IL-36a en un ambiente deficiente de IL-36RN, aumenta la severidad del fenotipo de piel y la letalidad neonatal

Se detectó elevado el IL-36R en la epidermis de 9 pacientes con placas, pero no en piel sana. La IL-36a se encontró fuertemente en la epidermis en las placas de los 10 pacientes, pero no piel no en la piel lesional. El IL-36Ra es expresado tanto en placas psoriáticas como en la piel sana de todos los pacientes.

Se realizó RT-PCR a IL-36R, IL-36a y IL-36Ra en 7 muestras de piel psoriática y no lesional; la mayor expresión de RNAm de las tres se encontró en las muestras psoriáticas
Blumberg et al.

Tabla 3. Evidencia experimental del papel de IL-36 en el síndrome DITRA 
mutación homocigota de c. [28C>T] en IL36RN, la cual causó p.[Arg10Ter]. Se procedió a la aféresis de granulocitos y monocitos, la cual fue efectiva, mejorando los síntomas de psoriasis pustulosa generalizada y disminuyendo los niveles séricos de IL-6 y de IL-8 ${ }^{(37)}$. El tratamiento anti-TNFa en pacientes con psoriasis resulta en la disminución de la expresión de agonistas de la IL-36, lo cual también se asocia con mejoría en los resultados clínicos ${ }^{(15)}$. Hay un reporte de una familia de tres hermanos y su madre, a quienes se les diagnosticó psoriasis pustulosa generalizada causada por DITRA, los cuales no tuvieron resultados satisfactorios con varios medicamentos, incluyendo la ciclosporina. Por esta razón, se decidió administrar infliximab en tres de los cuatro pacientes, así: $5 \mathrm{mg} / \mathrm{kg}$ el primer día, dos semanas y cuatro semanas después, y posteriormente, una vez cada ocho semanas como tratamiento de mantenimiento. Las lesiones se resolvieron rápidamente y no hubo recaída después de tres años de seguimiento; además, no se presentaron efectos secundarios aparentes ${ }^{(48)}$. También, se encontró el caso de un niño chino de ocho años con psoriasis pustulosa generalizada, a quien se le encontró mutación en el gen IL36RN y presentó falla terapéutica con la ciclosporina y que mejoró con infliximab ${ }^{(49)}$.

Recientemente, Arakawa inició estudios de cuatro pacientes con psoriasis pustulosa generalizada y mutaciones en el IL36RN, suministrándoles ustekinumab. Unos habían recibido previamente un tratamiento convencional para la psoriasis, anti-TNFa o ambos, sin resultados satisfactorios o con pérdida de la eficacia; otros no lo recibieron porque tenían contraindicaciones. Se obtuvieron remisiones sostenidas en los 44 meses de tratamiento y aún se encuentran bajo seguimiento ${ }^{(50)}$.

Hay hallazgos paradójicos en cuanto al anakinra, un antagonista recombinante del receptor de IL-1. Por un lado, en reportes de casos anecdóticos fue usado para el tratamiento de la psoriasis pustulosa generalizada o la acrodermatitis continua de Hallopeau, sin que resultara óptimo, posiblemente, por la evidencia experimental de que las vías de IL-1 e IL-36 contribuyen independientemente a la inflamación de la piel. Por otro lado, Hüffmeier, et al., y Rossi-Semerano, et al., reportaron que el anakinra fue efectivo en un caso de DITRA ${ }^{51,52)}$. También, existe un reporte de un paciente de 54 años con historia de psoriasis pustulosa, que había sido tratado con acitretín y ciclosporina sin mejoría. Posteriormente, se le inició infliximab con metotrexato, con lo que desarrolló neumonía por Pneumocystis jirovecii; después de esto, descubrieron una mutación heterocigota en el gen IL36RN, sugestiva de
DITRA, por lo que decidieron iniciar el tratamiento con 100 mg/diarios de anakinra, lo que resultó en una rápida mejoría de las lesiones, permaneciendo asintomático hasta después de doce meses de seguimiento ${ }^{(53)}$. Por lo tanto, actualmente, un tratamiento recomendable requiere agentes cuyo blanco específico sea la IL36, las vías comunes de señalización entre IL-1 y IL-36 ${ }^{(11,16)}$, o la proteína recombinante Il-36RN ${ }^{(29)}$. Por ahora, se ha propuesto recientemente un anticuerpo contra el receptor IL-36R, como una terapia potencial para la psoriasis causada por deficiencia del antagonista de dicho receptor.

Este tratamiento aún se encuentra en etapa experimental, pero, con base en los datos obtenidos en ratones e in vitro, parece ser prometedor para la psoriasis, especialmente, para la psoriasis pustulosa generalizada ${ }^{(54)}$.

\section{CONCLUSIONES}

Los avances en la comprensión de la inmunopatogénesis de las enfermedades cutáneas han permitido esclarecer las bases genéticas de enfermedades cutáneas graves, como la psoriasis pustulosa generalizada. El síndrome DITRA se debe a mutación del gen IL36RN y debe sospecharse en los pacientes con psoriasis pustulosa generalizada no asociada con placas de psoriasis vulgar. El tratamiento se enfoca en el uso de anti-TNF- $\alpha$, anti-IL-1 y, en el futuro, los antagonistas de IL-36 o de su receptor.

\section{REFERENCIAS}

1. Rubartelli A. Autoinflammatory diseases. Immunol Lett. 2014;161:226-30.

2. Masters SL, Simon A, Aksentijevich I, Kastner DL. Horror autoinflammaticus: The molecular pathophysiology of autoinflammatory disease. Annu Rev Immunol. 2009;27:621-68.

3. Moll M, Kuemmerle-Deschner JB. Inflammasome and cytokine blocking strategies in autoinflammatory disorders. Clin Immunol. 2013;147:242-75.

4. Günther S, Sundberg EJ. Molecular determinants of agonist and antagonist signaling through the IL-36 receptor. J Immunol. 2014;193:921-30.

5. Moghaddas F, Masters SL. Monogenic autoinflammatory diseases: Cytokinopathies. Cytokine. 2015;74:237-46.

6. Clavel G, Thiolat A, Boissier MC. Interleukin newcomers creating new numbers in rheumatology: IL-34 to IL-38. Joint Bone Spine. 2013;80:449- 
53.

7. Sánchez GA, de Jesús AA, Goldbach-Mansky R. Monogenic autoinflammatory diseases: Disorders of amplified danger sensing and cytokine dysregulation. Rheum Dis Clin North Am. 2013;39:701-34.

8. Gurung P, Kanneganti TD. Autoinflammatory skin disorders: The inflammasomme in focus. Trends Mol Med. 2016;22:545-64.

9. Picard C, Al-Herz W, Bousfiha A, Casanova JL, Chatila T, Conley ME, et al. Primary immunodeficiency diseases: An update on the classification from the International Union of Immunological Societies Expert Committee for Primary Immunodeficiency 2015. J Clin Immunol. 2015;35:696-726.

10. Abbas O, Itani S, Ghosn S, Kibbi AG, Fidawi G, Farooq M, et al. Acrodermatitis continua of Hallopeau is a clinical phenotype of DITRA: Evidence that it is a variant of pustular psoriasis. Dermatology. 2013;226:28-31.

11. Marrakchi S, Guigue P, Renshaw BR, Puel A, Pei XY, Fraitag S, et al. Interleukin-36-receptor antagonist deficiency and generalized pustular psoriasis. N Engl J Med. 2011;365:620-8.

12. Dinarello CA. Overview of the interleukin-1 family of ligands and receptors. Semin Immunol. 2013;25:389-93.

13. Boraschi D, Tagliabue A. The interleukin-1 receptor family. Semin Immunol. 2013;25:394-407.

14. Lamacchia C, Palmer G, Rodríguez E, Martin P, Vigne S, Seemayer CA, et al. The severity of experimental arthritis is independent of IL-36 receptor signaling. Arthritis Res Ther. 2013;15:R38.

15. Gresnigt MS, van de Veerdonk FL. Biology of IL-36 cytokines and their role in disease. Semin Immunol. 2013;25:458-65.

16. Blumberg H, Dinh H, Trueblood ES, Pretorius J, Kugler D, Weng N, et al. Opposing activities of two novel members of the IL-1 ligand family regulate skin inflammation. J Exp Med. 2007;204:2603-14.

17. Derer A, Groetsch B, Harre U, Böhm C, Towne J, Schett G, et al. Blockade of IL-36 receptor signaling does not prevent from TNF-induced arthritis. PLoS One. 2014;9:e101954.

18. Debets R, Timans JC, Homey B, Zurawski S, Sana TR, Lo S, et al. Two novel IL-1 family members, IL-1 delta and IL-1 epsilon, function as an antagonist and agonist of NF-kappa B activation through the orphan IL-1 receptor-related protein 2. J Immunol. 2001;167:1440-6.

19. Towne JE, Renshaw BR, Douangpanya J, Lipsky BP, Shen M, Gabel CA, et al. Interleukin-36 (IL-36) ligands require processing for full agonist (IL-36a,
IL-36 $\beta$, and IL-36Y) or antagonist (IL-36Ra) activity. J Biol Chem. 2011;286:42594-602.

20. Farooq M, Nakai H, Fujimoto A, Fujikawa H, Matsuyama A, Kariya N, et al. Mutation analysis of the IL36RN gene in 14 Japanese patients with generalized pustular psoriasis. Hum Mutat. 2013;34:17683.

21. Foster AM, Baliwag J, Chen CS, Guzmán AM, Stoll SW, Gudjonsson JE, et al. IL-36 promotes myeloid cell infiltration, activation, and inflammatory activity in skin. J Immunol. 2014;192:6053-61.

22. van de Veerdonk FL, Stoeckman AK, Wu G, Boeckermann AN, Azam T, Netea MG, et al. IL-38 binds to the IL-36 receptor and has biological effects on immune cells similar to IL-36 receptor antagonist. Proc Natl Acad Sci U S A. 2012;109:3001-5.

23. van de Veerdonk FL, Netea MG. New Insights in the immunobiology of IL-1 family members. Front Immunol. 2013;4:167.

24. Palomo J, Dietrich D, Martin P, Palmer G, Gabay C. The interleukin (IL)-1 cytokine family - Balance between agonists and antagonists in inflammatory diseases. Cytokine. 2015;76:25-37.

25. Dunn EF, Gay NJ, Bristow AF, Gearing DP, O'Neill LA, Pei XY. High-resolution structure of murine interleukin 1 homologue $\mathrm{IL}-1 \mathrm{~F}_{5}$ reveals unique loop conformations for receptor binding specificity. Biochemistry. 2003;42:10938-44.

26. Abbas AK, Lichtman AH, Pillai S. Inmunología celular y molecular. Octava edición. Barcelona: Elsevier España; 2015. p. 545

27. Naik HB, Cowen EW. Autoinflammatory pustular neutrophilic diseases. Dermatol Clin. 2013;31:40525.

28. Martin MU, Wesche H. Summary and comparison of the signaling mechanisms of the Toll/interleukin-1 receptor family. Biochim Biophys Acta. 2002;1592:265-80.

29. Sugiura K. The genetic background of generalized pustular psoriasis: IL36RN mutations and CARD14 gain-of-function variants. J Dermatol Sci. 2014;74:187-92.

30. Towne JE, Garka KE, Renshaw BR, Virca GD, Sims JE. Interleukin (IL)-1F6, IL-1F8, and IL-1F9 signal through IL-1Rrp2 and IL-1RAcP to activate the pathway leading to NF-kappaB and MAPKs. J Biol Chem. 2004;279:13677-88.

31. Vigne S, Palmer G, Lamacchia C, Martin P, Talabot-Ayer D, Rodríguez E, et al. IL-36R ligands are potent regulators of dendritic and T cells. Blood. 2011;118:5813-23.

32. Keermann M, Kõks S, Reimann E, Abram K, Erm T, 
Silm H, et al. Expression of IL-36 family cytokines and IL-37 but not IL-38 is altered in psoriatic skin. J Dermatol Sci. 2015;80:150-2.

33. Tortola L, Rosenwald E, Abel B, Blumberg H, Schäfer M, Coyle AJ, et al. Psoriasiform dermatitis is driven by IL-36-mediated DC-keratinocyte crosstalk. J Clin Invest. 2012;122:3965-76.

34. Carrier Y, Ma HL, Ramon HE, Napierata L, Small C, O'Toole M, et al. Inter-regulation of Th17 cytokines and the IL-36 cytokines in vitro and in vivo: Implications in psoriasis pathogenesis. J Invest Dermatol. 2011;131:2428-37.

35. Gabay C, Towne JE. Regulation and function of interleukin-36 cytokines in homeostasis and pathological conditions 2015; 97.

36. Kanazawa N, Nakamura T, Mikita N, Furukawa F. Novel IL36RN mutation in a Japanese case of early onset generalized pustular psoriasis. J Dermatol. 2013;40:749-51.

37. Tominaga C, Yamamoto M, Imai Y, Yamanishi K. A case of old age-onset generalized pustular psoriasis with a deficiency of IL-36RN (DITRA) treated by granulocyte and monocyte apheresis. Case Rep Dermatol. 2015;7:29-35.

38. Sugiura K, Takemoto A, Yamaguchi M, Takahashi $\mathrm{H}$, Shoda Y, Mitsuma T, et al. The majority of generalized pustular psoriasis without psoriasis vulgaris is caused by deficiency of interleukin-36 receptor antagonist. J Invest Dermatol. 2013;133:2514-21.

39. Li M, Han J, Lu Z, Li H, Zhu K, Cheng R, et al. Prevalent and rare mutations in IL-36RN gene in Chinese patients with generalized pustular psoriasis and psoriasis vulgaris. J Invest Dermatol. 2013;133:2637-9.

40. Li X, Chen M, Fu X, Zhang Q, Wang Z, Yu G, et al. Mutation analysis of the IL36RN gene in Chinese patients with generalized pustular psoriasis with/without psoriasis vulgaris. J Dermatol Sci. 2014;76:132-8.

41. Capon F. IL36RN mutations in generalized pustular psoriasis: Just the tip of the iceberg? J Invest Dermatol. 2013;133:2503-4.

42. Hussain S, Berki DM, Choon SE, Burden AD, Allen $\mathrm{MH}$, Arostegui JI, et al. IL36RN mutations define a severe autoinflammatory phenotype of generalized pustular psoriasis. J Allergy Clin Immunol. 2015;135:1067-70.e9.

43. Cowen EW, Goldbach-Mansky R. DIRA, DITRA, and new insights into pathways of skin inflammation: What's in a name? Arch Dermatol. 2012;148:381-4. 44. Johnston A, Xing X, Guzmán AM, Riblett M, Loyd
CM, Ward NL, et al. IL-1F5, -F6, -F8, and -F9: A novel IL-1 family signaling system that is active in psoriasis and promotes keratinocyte antimicrobial peptide expression. J Immunol. 2011;186:2613-22.

45. Ramadas RA, Ewart SL, Iwakura Y, Medoff BD, LeVine AM. IL-36a exerts pro-inflammatory effects in the lungs of mice. PLoS One. 2012;7:e45784.

46. Almeida de Jesús A, Goldbach-Mansky R. Monogenic autoinflammatory diseases: concept and clinical manifestations. Clin Immunol. 2013;147:15574 .

47. Setta-Kaffetzi N, Navarini AA, Patel VM, Pullabhatla V, Pink AE, Choon SE, et al. Rare pathogenic variants in IL36RN underlie a spectrum of psoriasis-associated pustular phenotypes. J Invest Dermatol. 2013;133:1366-9.

48. Sugiura K, Endo K, Akasaka T, Akiyama M. Successful treatment with infliximab of sibling cases with generalized pustular psoriasis caused by deficiency of interleukin-36 receptor antagonist. J Eur Acad Dermatol Venereol. 2015;29:2054-6.

49. Pan J, Qiu L, Xiao T, Chen HD. Juvenile generalized pustular psoriasis with IL36RN mutation treated with short-term infliximab. Dermatol Ther. 2016;29:164-7.

50. Arakawa A, Ruzicka T, Prinz JC. Therapeutic efficacy of interleukin 12/interleukin 23 blockade in generalized pustular psoriasis regardless of IL36RN mutation satus. JAMA Dermatol. 2016;152:825-8.

51. Hüffmeier U, Wätzold $M$, Mohr J, Schön MP, Mössner R. Successful therapy with anakinra in a patient with generalized pustular psoriasis carrying IL36RN mutations. Br J Dermatol. 2014;170:202-4.

52. Rossi-Semerano L, Piram M, Chiaverini C, De Ricaud D, Smahi A, Koné-Paut I. First clinical description of an infant with interleukin-36-receptor antagonist deficiency successfully treated with anakinra. Pediatrics. 2013;132:e1043-7.

53. Podlipnik S, de la Mora L, Alsina M, Mascaró JM Jr. Pneumocystis jirovecii pneumonia in a patient with pustular psoriasis with an IL-36RN deficiency treated with infliximab: Case report and review of the literature. Australas J Dermatol. 2017;58:e44-7.

54. Wolf J, Ferris LK. Anti-IL-36R antibodies, potentially useful for the treatment of psoriasis: A patent evaluation of WO2013074569. 2014;24:477-9. 